\title{
CONSTRUÇÃO DE ÍNDICES DE VALOR DE IMPORTÂNCIA DE ESPÉCIES PARA ANÁLISE FITOSSOCIOLÓGICA DE FLORESTA OMBRÓFILA ATRAVÉS DE ANÁLISE MULTIVARIADA
}

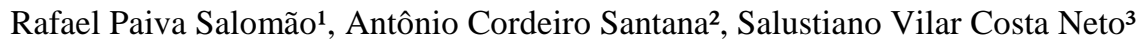 \\ 1Eng. Florestal, M.Sc., Coordenação de Botânica, MCTI/Museu Emílio Goeldi, Belém, PA - salomao@ museu-goeldi.br \\ ${ }^{2}$ Eng. Agrônomo, Dr., Instituto Sócio-Ambiental e de Recursos Hídricos, UFRA, Belém, PA - antonio.santana@ufra.edu.br \\ 3Biólogo, M.Sc., Instituto de Pesquisa Científica e Tecnológica do Estado do Amapá, IEPA, Macapá, AP - salustiano.neto@iepa.ap.gov.br
}

Recebido para publicação: 28/01/2011 - Aceito para publicação: 02/09/2011

\begin{abstract}
Resumo
Um importante instrumento da ecologia de comunidades é a análise multivariada, que trata todas as variáveis simultaneamente, sintetizando os dados e revelando a sua estrutura com a menor perda de informações possível. Objetivou-se neste trabalho construir, através da análise multivariada, dois índices fitossociológicos que envolvessem as mesmas variáveis do Índice de Valor de Importância (IVI), para comparação dos resultados entre estes índices, através do ranqueamento das espécies arbóreas amostradas em um inventário da Floresta Ombrófila Densa, em três categorias ecológicas previamente estabelecidas: predominância alta, intermediária e baixa. A adequação da análise fatorial foi determinada pelos testes Bartlett e KMO. O teste de Bartlett avaliou a significância geral da matriz de correlação, indicando que as correlações, em geral, são significantes ao nível de $1 \%$ de probabilidade. O teste $\mathrm{KMO}$ indicou que as variáveis estão correlacionadas e o modelo fatorial apresentou um nível bom de adequação aos dados. Estes resultados respaldaram o emprego da análise fatorial para a extração de fatores e a estimação dos escores fatoriais das espécies. O melhor índice entre os três foi aquele obtido através da análise fatorial, pelo método de componentes principais, que adicionou uma variável dummy para cada uma das variáveis envolvidas no modelo.

Palavras-chave: Espécie-chave; análise de componente principal (ACP); floresta tropical; fitossociologia; Amazônia.
\end{abstract}

\begin{abstract}
Construction of importance value index of species using multivariate analysis for phytosociological analysis of dense rain forest. An important tool for community ecology is multivariate analysis, which treats all variables simultaneously, summarizing data and revealing its structure with the smallest possible loss of information. This study aimed to develop, by multivariate analysis, two phytosociological indices derived from the same variables of the Importance Value Index (IVI), in order to compare such results between the indices by ranking of tree species sampled in a dense rain forest inventory, according to three previously established ecological categories: high, medium and low prevalence. The suitability of factor analysis was determined by Bartlett and KMO tests. Bartlett's test evaluated the overall significance of the correlation matrix indicating that correlations in general were significant at probability of $\mathrm{p}<0.01$. The KMO test indicated that the variables were correlated and the factorial model presented a good suitability level to the data. These results reinforce factor analysis using for factor extraction and estimating factor species scores. The best index of the three was obtained with factor analysis, which added a dummy variable to each variable in the model.

Keywords: Key specie; principal components analysis (PCA); rain forest; phytosociology; Amazon.
\end{abstract}

\section{INTRODUÇÃO}

A fitossociologia é um ramo da botânica que procura entender o conjunto de espécies que vivem e interagem em um determinado hábitat e os fatores ambientais e históricos relacionados a essa composição (RODRIGUES; GANDOLFI, 1996). O grande número de espécies vegetais, sobretudo nas florestas ombrófilas, dificulta a visualização da estrutura e de tendências pertinentes ao conjunto dos dados. Na comparação entre diferentes amostras, a estatística usual (univariada) não permite o tratamento 
das variáveis simultaneamente em uma mesma análise, sendo uma das soluções tratá-las através da análise multivariada, em que se podem envolver descritores sintéticos, como índices do somatório da abundância com a frequência e a dominância, riqueza, diversidade, entre outros.

Um importante instrumento da ecologia de comunidades é a análise multivariada, que trata todas as variáveis simultaneamente, sumarizando os dados e revelando a estrutura com a menor perda de informações possível (GAUCH, 1982; PIELOU, 1984). Diferentemente da estatística clássica, que possibilita o teste de hipótese, as análises multivariadas se prestam mais a investigar os dados e gerar hipóteses (GAUCH, 1982; OLIVEIRA-FILHO; RATTER, 1995). Costa (2003) ressalta que só recentemente as análises multivariadas tornaram-se instrumentos acessíveis, apesar de há muito conhecidas (GOODALL, 1954).

Muitos estudos em comunidades vegetais utilizam como base esse tipo de análise, tanto para agrupar amostras como para classificar tipos de formações, relacionar variáveis ambientais a diferenças na comunidade, definir prioridades para a conservação (TAGGART, 1994; OGUTU, 1996; LA ROI, 1992; KAPPELLE et al., 1995; OLIVEIRA-FILHO et al., 1994) e para a caracterização fitossociológica e pedológica em vegetação de cerrado (TOLEDO et al., 2009). Alguns estudos mais recentes com abordagem em análise multivariada foram desenvolvidos por Oliveira-Filho e Fontes (2000) e Scudeller et al. (2001) na Mata Atlântica do Sudeste brasileiro.

Objetivou-se neste trabalho construir, através da análise multivariada, dois índices fitossociológicos que envolvessem as mesmas variáveis do Índice de Valor de Importância (IVI), proposto por Curtis e McIntosh (1951), para posterior comparação dos resultados entre os índices, através do ranqueamento das espécies amostradas em um inventário da Floresta Ombrófila Densa, em três categorias ecológicas previamente estabelecidas: predominância alta, intermediária e baixa. Adicionalmente, foi feita também uma breve análise e discussão da composição florística e da estrutura da floresta estudada.

Considerando-se, neste estudo, que o IVI não é uma ferramenta adequada para a seleção de espécies-chave a serem plantadas nas áreas anuais de restauração florestal, após a lavra do minério, adotou-se a hipótese de que um índice obtido através da análise multivariada, empregando-se as mesmas variáveis do IVI, torna-se mais apropriado para essa seleção.

A construção dos índices tem por objetivo subsidiar e auxiliar na tomada de decisão de quais espécies arbóreas serão prioritárias (espécies-chave) nos trabalhos de restauração florestal em áreas de preservação permanente (APP), áreas de reserva legal (ARL), áreas de mineração a céu aberto, entre outras, posto que, com o advento do cadastro ambiental rural, os proprietários e detentores de posses são obrigados a recuperar o passivo ambiental em suas terras (APP e/ou ARL).

\section{MATERIAL E MÉTODO}

\section{Área de estudo}

O inventário fitossociológico foi realizado em uma área de 3.750 ha, denominada platô Monte Branco, com altitude média de $180 \mathrm{~m}$, localizada na Floresta Nacional (FLONA) Saracá Taquera/ICMBIO, no distrito de Porto Trombetas $\left(1^{\circ} 21^{\prime} \mathrm{S}-56^{\circ} 22^{\prime} \mathrm{W}\right)$, município de Oriximiná, estado do Pará, Brasil. Nessa Flona, existem 23 platôs compostos por terrenos planos ou pouco ondulados, elevados, cortados por vales neles encaixados, com ocorrência de bauxita no subsolo, onde a Mineração Rio do Norte S.A. (MRN) desenvolve um projeto mínero-industrial de bauxita. Esses platôs são bem definidos, têm os topos planos, achatados, com altitudes entre $150 \mathrm{~m}$ e $200 \mathrm{~m}$ em relação ao nível do mar.

$\mathrm{Na}$ bacia amazônica, os depósitos de bauxita são associados à série Barreiras do Terciário, constituídos de arenitos, siltitos e, ocasionalmente, conglomerados. As lateritas são encontradas no topo dos platôs, fortemente dissecados pela erosão, remanescentes do Peneplano Terciário, que se estendem ao longo do lado nordeste do rio Amazonas, desde as vizinhanças de Oriximiná até Jardilândia, no rio Jari (LAPA, 2000).

A área acha-se inserida na região da Floresta Ombrófila Densa, sub-região dos baixos platôs da Amazônia, domínio da Floresta Densa Submontana, cuja fitofisionomia refere-se à floresta localizada principalmente nos platôs terciários e terraços antigos e recentes, apresentando-se em dois estratos distintos: um emergente e outro uniforme (PROJETO RADAM, 1976). As principais espécies que caracterizam o estrato emergente são: Dinizia excelsa Ducke ('angelim-pedra'), Bertholletia excelsa H. \& B. ('castanheira') e Cedrelinga catanaeformis Ducke ('cedrorana'). $\mathrm{O}$ estrato uniforme é caracterizado por espécies dos gêneros Manilkara Adans. ('maçarandubas'), Protium Burnm. F. ('breus') e Pouteria Aubl. ('abius'). 
O clima da região é do tipo Af, de acordo com a classificação climática de Köppen (1928), com A caracterizando clima megatérmico tropical, com temperatura média do mês mais frio superior a $18{ }^{\circ} \mathrm{C}$, estação de inverno ausente e forte precipitação anual (superior à evapotranspiração potencial anual), e $\underline{\mathrm{f}}$ informando que o clima é úmido, com ocorrência de precipitação em todos os meses do ano e inexistência de estação seca definida. De acordo com o Instituto Nacional de Meteorologia (INMET, 2006), a precipitação pluviométrica média anual varia de 2.500 a $3.000 \mathrm{~mm}$, com médias anuais de temperatura, precipitação pluviométrica, umidade relativa e insolação anual de, respectivamente, $26{ }^{\circ} \mathrm{C}, 2.197 \mathrm{~mm}$, $81 \%$ e 2.026 horas. Informações mais detalhadas da área incluindo clima, solo, geologia, geomorfologia e vegetação foram apresentadas por Salomão et al. (2007a).

\section{Processo de amostragem}

O processo de amostragem utilizado no inventário florestal foi baseado na amostragem sistemática, também conhecida como seleção mecânica, em que as unidades amostrais são selecionadas segundo um rígido e predeterminado esquema que procura cobrir toda a população. A vantagem mais importante da amostra sistemática, quando aplicada em florestas primárias, é que as unidades amostrais se distribuem mais uniformemente na população, originando uma maior representatividade. Dessa forma, torna-se mais eficiente para detectar tendência ou concentração de certas características, assim como é mais precisa na observação das variáveis tipológicas (CAMPOS; LEITE, 2002).

A unidade amostral empregada possuía a forma retangular, com $10 \mathrm{~m}$ de largura por $250 \mathrm{~m}$ de comprimento $(0,25 \mathrm{ha})$, totalizando 301 parcelas $(75,25 \mathrm{ha})$, onde foram registradas, identificadas e mensuradas todas as árvores, cipós e palmeiras com diâmetro à altura do peito (DAP) $\geq 10 \mathrm{~cm}$. As espécies registradas foram identificadas e classificadas pelo sistema de Cronquist (1981). Aquelas que apresentaram material fértil tiveram o mesmo coletado e depositado no herbário do Museu Emílio Goeldi (MCTI).

\section{Estrutura horizontal}

A estrutura horizontal da floresta foi caracterizada através das variáveis fitossociológicas, incluídas na análise fatorial, como Abundância (ABR), Frequência (FRR) e Dominância (DOR) relativa das espécies registradas nas parcelas da amostra. Essas variáveis foram utilizadas na definição de um Índice de Fitossociologia Horizontal (IFH), construído a partir da análise fatorial descrita na próxima seção, para ser comparado com o IVI da espécie, proposto por Curtis e McIntosh (1951). Adicionalmente, definiu-se, para cada uma dessas variáveis, uma variável qualitativa (variável dummy, variável binária ou variável artificial), para captar a influência das espécies em que pelo menos 50\% dos valores das variáveis envolvidas (abundância, frequência e dominância) constavam na amostra.

O IVI da espécie, proposto por Curtis e McIntosh (1951), é obtido pelo somatório dos valores relativos da abundância, frequência e dominância dos indivíduos de cada espécie registrada na amostra.

$$
I V I=A B R+F R R+D O R
$$

em que: $\mathrm{ABR}=\mathrm{n}^{\mathrm{o}}$ de indivíduos da espécie $/ \mathrm{n}^{\mathrm{o}}$ total de indivíduos;

$\mathrm{FRR}=\mathrm{n}^{\mathrm{o}}$ de parcelas em que ocorre a espécie / soma do $\mathrm{n}^{\circ}$ de parcelas em que ocorre a espécie, para todas as espécies;

DOR = área seccional total dos indivíduos da espécie / área seccional total de todos os indivíduos das espécies.

\section{Modelo analítico}

A análise fatorial foi utilizada pioneiramente para determinar as relações conjuntas de variáveis indicadoras da fitossociologia de uma floresta ombrófila da Amazônia e construir um novo índice comparável ao índice de valor de importância a partir da combinação dos fatores comuns representativos das variáveis (ABR, FRR e DOR) definidas no parágrafo anterior.

Inicialmente, a análise foi orientada para confirmar três fatores correspondentes a essas três variáveis e representar a variância total dos dados. Em seguida, para assegurar a participação das espécies relevantes na amostra, o modelo foi reestimado com a presença das variáveis (ABR-VD, FRR-VD e DOR-VD).

De modo geral, emprega-se a análise fatorial para analisar a estrutura das inter-relações (correlações) entre um grande número de variáveis quantitativas e/ou qualitativas e definir um conjunto menor de dimensões latentes ou fatores comuns, com o objetivo de facilitar a compreensão por meio de 
adequada representação da estrutura da nuvem de dados (SANTANA, 2005; 2008). Assim, foi possível identificar e isolar as dimensões da estrutura dos dados e então determinar o grau em que cada variável foi explicada por cada dimensão latente ou fator comum.

O modelo geral de análise fatorial pode ser apresentado na forma matricial, como em Dillon e Goldstein (1984):

$$
X=\alpha f+\varepsilon
$$

em que: $\mathrm{X}=$ é o p-dimensional vetor transposto das variáveis observáveis, denotado por $X=\left(x_{1}, x_{2}, \ldots\right.$, $\left.x_{p}\right)$

$f=$ é o q-dimensional vetor transposto de variáveis não observáveis ou variáveis latentes chamadas de fatores comuns, denotado por, $\boldsymbol{f}=\left(f_{1}, f_{2}, \ldots, f_{q}\right)$, sendo que $\mathrm{q}<\mathrm{p}$;

$\varepsilon=$ é o p-dimensional vetor transposto de variáveis aleatórias ou fatores únicos, $\varepsilon=\left(e_{1}, e_{2}, \ldots\right.$, $\left.e_{p}\right)$

$\alpha=$ é a matriz $(\mathrm{p}, \mathrm{q})$ de constantes desconhecidas, chamadas de cargas fatoriais. No modelo de análise fatorial, pressupõe-se que os fatores específicos são ortogonais entre si e com todos os fatores comuns, ou seja, a covariância entre eles é zero. Especificamente, $E(\varepsilon)=E(\boldsymbol{f})=0$ e $C o v$ $(\varepsilon, f)=0$.

A estrutura inicial utilizada para determinar a matriz de cargas fatoriais, em geral, não é definitiva, por não assumirem uma adequada posição ortogonal; logo, pode não gerar um padrão significativo para as cargas das variáveis. A confirmação ou não dessa estrutura ortogonal inicial pode ser feita por meio de vários métodos de rotação dos fatores (DILLON; GOLDSTEIN, 1984; JOHNSON; WICHERN, 1992). Neste trabalho, utilizou-se o método varimax de rotação ortogonal dos fatores, pela simplicidade e os bons resultados que apresenta. O método varimax é um processo em que os eixos de referência dos fatores são rotacionados em torno da origem até que alguma outra posição ortogonal seja alcançada. Nesse processo, ocorre uma redistribuição da variância dos primeiros fatores para os demais, até se atingir um padrão fatorial mais simples e teoricamente mais significativo (HAIR et al., 2005; SANTANA; SANTANA, 2004; SANTANA, 2005).

A escolha dos fatores foi realizada por meio da técnica de raiz latente. Essa técnica parte do princípio de que qualquer fator individual, para ser extraído, deve explicar a variância de pelo menos uma variável para que seja mantido para interpretação. Cada variável contribui com um valor 1 da variância total ou do autovalor total. Com efeito, apenas os fatores que têm raízes latentes ou autovalores maiores que 1 são considerados significantes, e os demais fatores com autovalores menores do que 1 são considerados insignificantes e descartados (HAIR et al., 2005; MINGOTI, 2005; SANTANA, 2007; 2008). A matriz de cargas fatoriais, que mede a correlação entre os fatores comuns e as variáveis observáveis, é determinada a partir da matriz de correlação, conforme Dillon e Goldstein (1984).

Para a definição do IFH, procedeu-se, conforme sugerido por Santana $(2007 ; 2008)$, à estimação da matriz de escores fatoriais após a rotação ortogonal da estrutura fatorial inicial. O escore fatorial, por definição, situa cada observação no espaço dos fatores comuns. Para cada fator $\boldsymbol{f}_{j}$, o i-ésimo escore fatorial extraído é definido por $F_{i j}$, expresso por meio de uma combinação linear da forma descrita em Dillon e Goldstein (1984):

$$
F_{i j}=b_{1} x_{i 1}+b_{2} x_{i 2}+\ldots+b_{p} x_{i p}
$$

em que: $i=1,2, \ldots, n ; \quad j=1,2, \ldots, p$;

$b_{i}$ são os coeficientes de regressão estimados para os $n$ escores fatoriais comuns;

$x_{i j}$ são as $n$ observações das $p$ variáveis observáveis, padronizadas pelo método Z-score para apresentar uma distribuição com média 0 e desvio igual a 1.

$F_{i j}$ não é observável, mas pode ser estimada por meio das técnicas de análise fatorial, utilizandose a matriz de observações do vetor $\mathrm{x}$ de variáveis observáveis.

Para a construção do IFH, o escore fatorial $\left(F_{i}\right)$ foi padronizado pela amplitude para se obter valores positivos dos escores originais para variar entre 0 e $1\left(F P_{i}\right)$ e permitir a hierarquização das espécies. A fórmula matemática é a seguinte:

$$
F P_{i}=\left(\frac{F_{i}-F_{\text {min }}}{F_{\text {max }}-F_{\text {min }}}\right)
$$


em que: Fmin e Fmax são os valores máximo e mínimo observados para os escores fatoriais associados às variáveis (ABR, FRR, DOR, ABR-VD, FRR-VD e DOR-VD) das 745 espécies.

O IFH foi definido como uma combinação linear desses escores fatoriais e a proporção da variância explicada por cada fator, em relação à variância explicada pelos fatores comuns, sem ou com a variável dummy $(k)$. A expressão matemática é dada por (SANTANA, 2007; 2008):

$$
I F H_{i, k}=\sum_{j=1}^{q}\left(\frac{\lambda_{j}}{\sum_{j} \lambda_{j}} F P_{i j}\right)
$$

em que: $\lambda$ é a variância explicada por cada fator;

$\Sigma \lambda$ é a soma total da variância explicada pelo conjunto de fatores comuns extraídos.

$\mathrm{Na}$ análise fatorial, as variáveis explicativas $X_{i}(i=1,2, \ldots, n)$ são quantitativas e qualitativas. A variável de natureza qualitativa incluída na análise teve a missão de identificar a importância de algumas espécies na amostra. Para isso, atribuiu-se valor 1 para indicar a presença da característica de interesse e valor 0 para revelar a ausência do fenômeno.

Os fenômenos analisados cuja variável dummy foi igual a 1 referem-se àquelas espécies que, ocupando as primeiras posições em um rol decrescente de abundância, frequência e dominância absolutas, perfazem, em conjunto ou individualmente, mais de $50 \%$ do valor total de cada uma dessas variáveis. Por exemplo: a abundância absoluta total foi de $x$ indivíduos; após a hierarquização decrescente das espécies, do maior para o menor valor dessa variável, procedeu-se à soma do número de indivíduos de cada espécie até se atingir $50 \%$ do total de $x$, e as espécies aí incluídas receberam valor 1 na variável dummy, enquanto as demais, o valor zero; procedimento análogo foi feito para a frequência e a dominância.

Para aferir a adequação do método à amostra de dados, foram aplicados os dois testes: (i) teste de Kaiser-Meyer-Olkin (KMO), que se baseia no princípio de que a inversa da matriz de correlação se aproxima da matriz diagonal, comparando, para tanto, as correlações entre as variáveis observáveis (HAIR et al., 2005); e (ii) teste de esfericidade de Bartlett, que avalia a significância geral da matriz de correlação, ou seja, testa a hipótese nula de que a matriz de correlação é uma matriz identidade (DILLON; GOLDSTEIN, 1984; GAMA et al., 2007). Os resultados foram obtidos por meio do software SPSS Statistics $17.0^{\mathrm{TM}}$.

\section{Ranqueamento das espécies}

Para facilitar a interpretação dos resultados, foram estabelecidos três intervalos de predominância para os três índices (IFH sem e com a variável dummy e o IVI), obtidos pela diferença entre o maior e o menor valor calculado para cada índice, dividida por 3, aqui denominada amplitude de ranqueamento (AR).

Espécies de alta predominância foram aquelas situadas no intervalo entre o maior valor do respectivo índice calculado subtraído da amplitude de ranqueamento, enquanto as espécies com predominância baixa foram aquelas que se encontravam no intervalo entre o menor valor do respectivo índice, acrescido da amplitude de ranqueamento. As espécies cujos valores situavam-se entre o valor mínimo do índice mais a amplitude de ranqueamento e o valor máximo do índice menos a amplitude de ranqueamento foram classificadas como de predominância intermediária.

\section{RESULTADOS}

\section{Florística}

Foram registrados 36.546 indivíduos (DAP $\geq 10 \mathrm{~cm}$ ), totalizando 745 espécies, distribuídas em 61 famílias nas 301 parcelas de 0,25 ha cada, totalizando 75,25 ha de vegetação amostrada (intensidade amostral de 2,01\%) do inventário florestal em 3.750 ha do platô Monte Branco.

A maior riqueza de espécies foi apresentada por Sapotaceae (93 espécies), Mimosaceae (52), Lauraceae (43), Fabaceae (38), Annonaceae (35), Myrtaceae (34), Chrysobalanaceae (33), Burseraceae e Caesalpiniaceae (30 espécies cada), Moraceae (28) e Lecythidaceae, com 27 espécies; 11 famílias (ou $18 \%$ do total) ocorreram com apenas uma espécie. Considerando-se, doravante, Leguminosae como o somatório da variável em questão, referente às famílias Caesalpiniaceae, Mimosaceae e Fabaceae, ela seria, neste caso, a de maior riqueza, com 120 espécies. 
Sapotaceae foi a família que apresentou também a maior abundância (5.410 indivíduos), seguindo-se Violaceae (4.467), Lecythidaceae (3.521), Annonaceae (2.423), Mimosaceae (1.790) e Burseraceae (1.756), que responderam por 53,0\% (ou 19.367 espécimes) do total. Considerando-se Leguminosae, ela seria a terceira família mais abundante, com 3.813 indivíduos.

As espécies de maior abundância na amostragem foram Rinorea racemosa (Mart.) Kuntze, com 1.767 indivíduos, Rinorea guianensis Aubl. (1.762), Eschweilera coriacea (DC.) S.A.Mori (1.271), Geissospermum sericeum (Benth.) Hook. (788), Eschweilera amazonica R. Knuth (787), Oenocarpus bacaba Mart. (672), Rionorea riana Kuntze (662) e Guatteria olivacea R.E. Fries (589). Essas oito espécies (ou 1,1\% do total) ocorreram com 8.298 do total de indivíduos (ou 22,7\% do total); no outro extremo, 137 espécies (ou 18,4\% do total) ocorreram com apenas um indivíduo.

Nenhuma espécie ocorreu em todas as 301 unidades amostrais, consequentemente nenhuma apresentou $100 \%$ de frequência. Somente Eschweilera coriacea, Rinorea racemosa, Geissospermum sericeum e Rinorea guianensis estiveram próximas da frequência máxima, ocorrendo em 294 (97,7\% do total), $288(95,7 \%), 284(94,4 \%)$ e $272(90,4 \%)$ parcelas, respectivamente; no outro extremo, 148 espécies (ou 19,9\% do total) ocorreram em apenas uma parcela da amostragem.

\section{Índices fitossociológicos}

Índice fitossociológico horizontal

A adequação da análise fatorial foi determinada pelos testes Bartlett e KMO. O teste de Bartlett avaliou a significância geral da matriz de correlação e apresentou um valor de 1.339,73, indicando que as correlações, em geral, são significantes ao nível de $1 \%$ de probabilidade, ou seja, a matriz de correlação não é diagonal. O teste $\mathrm{KMO}$, da ordem de 0,709 , indicou que as variáveis estão correlacionadas e o modelo fatorial apresentou um nível médio de adequação aos dados (HAIR et al., 2005). Estes resultados respaldam o emprego da análise fatorial para a extração de fatores e a estimação dos escores fatoriais.

A solução rotacionada confirmou a extração dos três fatores predeterminados. Como o número de fatores é igual ao número de variáveis, a variância específica é nula, pois os fatores explicam $100 \%$ da variância total da nuvem de dados (Tabela 1). Na referida tabela, a penúltima linha contém o resultado para a variância total explicada por cada uma das componentes (autovalor) e na última linha estão os percentuais da variância explicada pelas respectivas componentes, em que a componente 1 explicou $35,329 \%$ da variância total, a componente 2 explicou 32,632\% e a componente 3 explicou 32,039\%.

Tabela 1. Matriz de cargas fatoriais $(\alpha)$ rotacionadas pelo método varimax com respectivas variáveis dos componentes obtidos (IFH).

Table 1. Matrix of factor loadings $(\alpha)$ rotated by varimax method with their component variables obtained (IFH).

\begin{tabular}{lcccc}
\hline \multirow{2}{*}{ Variável } & \multicolumn{3}{c}{ Componentes } & \multirow{2}{*}{ Comunalidade } \\
\cline { 2 - 4 } & $\mathbf{1}$ & $\mathbf{2}$ & $\mathbf{3}$ & 1,000 \\
Abundância relativa - ABR & 0,324 & 0,845 & 0,426 & 1,000 \\
Frequência relativa - FRR & 0,331 & 0,434 & 0,838 & 1,000 \\
Dominância relativa - DOR & 0,919 & 0,277 & 0,279 & 3,000 \\
Autovalor & 1,060 & 0,979 & 0,961 & 100,00 \\
Variância explicada (\%) & 35,329 & 32,632 & 32,039 & \\
\hline
\end{tabular}

A comunalidade, que reflete o grau em que cada variável é explicada pelas três componentes, consta na coluna quatro da tabela 1 . A penúltima linha da tabela representa os autovalores, que indicam a importância relativa de cada fator na explicação da variância total. A soma dos autovalores é igual a 3,0, que corresponde à variância total dos dados da amostra. Portanto, o índice IFH foi construído com base em $100 \%$ da variância total dos dados, sugerindo uma plena representatividade do fenômeno estudado.

O índice IFH calculado para todas as 745 espécies variou de 0,337\% a 0,114\%, gerando uma amplitude de 0,223\% que, dividida por 3, resultou numa Amplitude de Ranqueamento (AR) igual a 0,074\%. De acordo as categorias preestabelecidas, foram então geradas as classes de alta predominância (de $0,337 \%$ a 0,263\%), predominância intermediária (de 0,262\% a 0,187\%) e baixa predominância (de $0,188 \%$ a $0,114 \%$ ). Seis espécies foram classificadas, através desse índice, como de predominância alta, 42 como intermediárias, sendo as demais 697 classificadas como de baixa predominância (Tabela 2). 
Tabela 2. Número de espécies e percentual relativo por índice fitossociológico e por categoria de predominância ecológica das espécies amostradas.

Table 2. Number of species and relative percentage by phytosociological index and ecological dominance category of sampled species.

\begin{tabular}{lccccc}
\hline \multirow{2}{*}{ Índice } & \multicolumn{3}{c}{ Categoria de predominância ecológica da espécie } & \multirow{2}{*}{ Total de } \\
\cline { 2 - 5 } & $\begin{array}{c}\mathbf{A} \\
\text { Alta }\end{array}$ & $\begin{array}{c}\mathbf{B} \\
\text { Intermediária }\end{array}$ & $\mathbf{A + B}$ & $\begin{array}{c}\mathbf{C} \\
\text { Baixa }\end{array}$ & espécies \\
\hline IFH & $6(0,81 \%)$ & $42(5,64 \%)$ & $48(6,44 \%)$ & $697(93,56 \%)$ & $745(100 \%)$ \\
IFH-VD & $29(3,89 \%)$ & $52(6,98 \%)$ & $81(10,87 \%)$ & $664(89,13 \%)$ & $745(100 \%)$ \\
IVI & $4(0,54 \%)$ & $6(0,81 \%)$ & $10(1,34 \%)$ & $735(98,66 \%)$ & $745(100 \%)$ \\
\hline
\end{tabular}

As seis espécies com alta predominância (Geissospermum sericeum, Eschweilera coriacea, Minquartia guanensis Aubl., Eschweilera amazonica, Hevea guianensis Aubl. e Oenocarpus bacaba) e as 42 classificadas como intermediárias, mais as 33 de maiores valores na categoria de baixa predominância encontram-se relacionadas na tabela $3 \mathrm{com}$ seus respectivos valores do índice.

Tabela 3. Relação das espécies Classificadas nas Categorias (CAT) de predominância ecológica A (alta) e B (intermediária), através do índice fitossociológico com variável dummy (IFH-VD), e respectivos valores do índice fitossociológico horizontal (IFH) e índice de valor de importância (IVI).

Table 3. List of species classified in categories (CAT) ecological predominance A (high) and B (intermediate), by phytosociological index with dummy variable (IFH-VD), and their horizontal phytosociological index values (IFH) and importance value index (IVI).

\begin{tabular}{|c|c|c|c|c|c|c|c|}
\hline \multirow{2}{*}{ Nome científico } & \multirow{2}{*}{ Família } & \multicolumn{2}{|c|}{ IVI } & \multicolumn{2}{|c|}{ IFH } & \multicolumn{2}{|c|}{ IFH-VD } \\
\hline & & $(\%)$ & CAT & $(\%)$ & CAT & $(\%)$ & CAT \\
\hline Geissospermum sericeum & Apocynaceae & 2,810 & $\mathrm{~A}$ & 0,337 & $\mathrm{~A}$ & 0,242 & $\mathrm{~A}$ \\
\hline Oenocarpus bacaba & Arecaceae & 1,196 & $\mathrm{~B}$ & 0,268 & A & 0,240 & A \\
\hline Eschweilera coriacea & Lecythidaceae & 3,408 & $\mathrm{~A}$ & 0,321 & A & 0,239 & $\mathrm{~A}$ \\
\hline Eschweilera amazonica & Lecythidaceae & 1,432 & $\mathrm{~B}$ & 0,271 & A & 0,239 & $\mathrm{~A}$ \\
\hline Minquartia guanensis & Olacaceae & 1,207 & B & 0,272 & A & 0,238 & A \\
\hline Hevea guianensis & Euphorbiaceae & 1,348 & B & 0,271 & A & 0,237 & A \\
\hline Pouteria minutiflora & Sapotaceae & 0,979 & $\mathrm{C}$ & 0,257 & B & 0,237 & A \\
\hline Candolleodendron brachystachyum & Fabaceae & 0,850 & $\mathrm{C}$ & 0,254 & B & 0,237 & A \\
\hline Guatteria olivacea & Annonaceae & 1,111 & $\mathrm{C}$ & 0,244 & B & 0,235 & A \\
\hline Rinorea racemosa & Violaceae & 2,361 & A & 0,234 & B & 0,235 & A \\
\hline Eschweilera grandiflora & Lecythidaceae & 0,874 & $\mathrm{C}$ & 0,239 & B & 0,235 & A \\
\hline Rinorea guianensis & Violaceae & 2,726 & A & 0,237 & B & 0,234 & A \\
\hline Endopleura uchi & Humiriaceae & 0,826 & $\mathrm{C}$ & 0,234 & B & 0,233 & A \\
\hline Ocotea percurrens & Lauraceae & 0,736 & $\mathrm{C}$ & 0,224 & B & 0,232 & A \\
\hline Pouteria resinifera & Sapotaceae & 0,880 & $\mathrm{C}$ & 0,228 & B & 0,232 & A \\
\hline Ecclinusa guianensis & Sapotaceae & 0,974 & $\mathrm{C}$ & 0,233 & B & 0,232 & A \\
\hline Licania impressa & Chrysobalanaceae & 1,166 & B & 0,237 & B & 0,232 & A \\
\hline Pouteria guianensis & Sapotaceae & 0,638 & $\mathrm{C}$ & 0,211 & B & 0,230 & A \\
\hline Pouteria eugeniifolia & Sapotaceae & 0,644 & $\mathrm{C}$ & 0,208 & $\mathrm{~B}$ & 0,229 & $\mathrm{~A}$ \\
\hline Goupia glabra & Celastraceae & 0,887 & $\mathrm{C}$ & 0,217 & $\mathrm{~B}$ & 0,229 & A \\
\hline Inga alba & Mimosaceae & 0,597 & $\mathrm{C}$ & 0,203 & B & 0,229 & A \\
\hline Pouteria peruviensis & Sapotaceae & 0,699 & $\mathrm{C}$ & 0,204 & B & 0,229 & A \\
\hline Eschweilera atropetiolata & Lecythidaceae & 0,800 & $\mathrm{C}$ & 0,206 & B & 0,229 & A \\
\hline Protium hebetatum & Burseraceae & 0,779 & $\mathrm{C}$ & 0,239 & $\mathrm{~B}$ & 0,204 & A \\
\hline Microppolis williamii & Sapotaceae & 0,632 & $\mathrm{C}$ & 0,232 & B & 0,203 & A \\
\hline Theobroma glaucum & Sterculiaceae & 0,586 & $\mathrm{C}$ & 0,230 & B & 0,203 & A \\
\hline Zygia racemosa & Mimosaceae & 0,657 & $\mathrm{C}$ & 0,232 & B & 0,203 & A \\
\hline Licania octandra & Chrysobalanaceae & 0,671 & $\mathrm{C}$ & 0,231 & B & 0,203 & A \\
\hline Unonopsis duckei & Annonaceae & 0,607 & $\mathrm{C}$ & 0,229 & $\mathrm{~B}$ & 0,203 & A \\
\hline
\end{tabular}




\begin{tabular}{|c|c|c|c|c|c|c|c|}
\hline Laetia procera & Flacourtiaceae & 0,576 & $\mathrm{C}$ & 0,201 & B & 0,202 & $\mathrm{~B}$ \\
\hline Matisia ochrocalyx & Bombacaceae & 0,555 & $\mathrm{C}$ & 0,226 & $\mathrm{~B}$ & 0,202 & $\mathrm{~B}$ \\
\hline Onychopetalum amazonicum & Annonaceae & 0,650 & $\mathrm{C}$ & 0,229 & B & 0,202 & $\mathrm{~B}$ \\
\hline Lecythis holcogyne & Lecythidaceae & 0,651 & $\mathrm{C}$ & 0,200 & B & 0,202 & $\mathrm{~B}$ \\
\hline Protium amazonicum & Burseraceae & 0,628 & $\mathrm{C}$ & 0,222 & B & 0,201 & B \\
\hline Swartzia polyphylla & Fabaceae & 0,756 & $\mathrm{C}$ & 0,202 & B & 0,201 & $\mathrm{~B}$ \\
\hline Swartzia recurva & Fabaceae & 0,592 & $\mathrm{C}$ & 0,193 & B & 0,201 & B \\
\hline Pouteria prancei & Sapotaceae & 0,677 & $\mathrm{C}$ & 0,219 & B & 0,201 & B \\
\hline Virola calophylla & Myristicaceae & 0,492 & $\mathrm{C}$ & 0,216 & B & 0,201 & B \\
\hline Protium apiculatum & Burseraceae & 0,552 & $\mathrm{C}$ & 0,216 & B & 0,201 & $\mathrm{~B}$ \\
\hline Couepia longipendula & Chrysobalanaceae & 0,557 & $\mathrm{C}$ & 0,214 & $\mathrm{~B}$ & 0,200 & $\mathrm{~B}$ \\
\hline Chimarrhis turbinata & Rubiaceae & 0,491 & $\mathrm{C}$ & 0,184 & $\mathrm{C}$ & 0,200 & B \\
\hline Iryanthera lancifolia & Myristicaceae & 0,424 & $\mathrm{C}$ & 0,181 & $\mathrm{C}$ & 0,200 & $\mathrm{~B}$ \\
\hline Brosimum rubescens & Moraceae & 0,454 & $\mathrm{C}$ & 0,182 & $\mathrm{C}$ & 0,200 & B \\
\hline Pouteria krukovii & Sapotaceae & 0,520 & $\mathrm{C}$ & 0,209 & $\mathrm{~B}$ & 0,199 & $\mathrm{~B}$ \\
\hline Pouteria petiolata & Sapotaceae & 0,533 & $\mathrm{C}$ & 0,208 & $\mathrm{~B}$ & 0,199 & $\mathrm{~B}$ \\
\hline Pseudopiptadenia suaveolens & Mimosaceae & 0,459 & $\mathrm{C}$ & 0,178 & $\mathrm{C}$ & 0,199 & $\mathrm{~B}$ \\
\hline Casearia javitensis & Flacourtiaceae & 0,394 & $\mathrm{C}$ & 0,202 & $\mathrm{~B}$ & 0,199 & B \\
\hline Dipleryx odorata & Fabaceae & 0,627 & $\mathrm{C}$ & 0,182 & $\mathrm{C}$ & 0,199 & B \\
\hline Tetragastris panamensis & Burseraceae & 0,507 & $\mathrm{C}$ & 0,200 & $\mathrm{~B}$ & 0,198 & $\mathrm{~B}$ \\
\hline Pouteria clandantha & Sapotaceae & 0,531 & $\mathrm{C}$ & 0,199 & $\mathrm{~B}$ & 0,198 & $\mathrm{~B}$ \\
\hline Guatteria umbonata & Annonaceae & 0,456 & $\mathrm{C}$ & 0,188 & $\mathrm{C}$ & 0,196 & $\mathrm{~B}$ \\
\hline Rinorea riana & Violaceae & 0,918 & $\mathrm{C}$ & 0,177 & $\mathrm{C}$ & 0,195 & B \\
\hline Eschweilera eperuatorum & Lecythidaceae & 0,458 & $\mathrm{C}$ & 0,174 & $\mathrm{C}$ & 0,194 & $\mathrm{~B}$ \\
\hline Bocageopsis multiflora & Annonaceae & 0,386 & $\mathrm{C}$ & 0,191 & $\mathrm{~B}$ & 0,171 & $\mathrm{~B}$ \\
\hline Sclerolobium chrysophyllum & Caesalpiniaceae & 0,453 & $\mathrm{C}$ & 0,191 & $\mathrm{~B}$ & 0,171 & $\mathrm{~B}$ \\
\hline Virola michelii & Myristicaceae & 0,425 & $\mathrm{C}$ & 0,190 & $\mathrm{~B}$ & 0,170 & $\mathrm{~B}$ \\
\hline Tapura amazonica & Dichapetalaceae & 0,335 & $\mathrm{C}$ & 0,184 & $\mathrm{C}$ & 0,170 & $\mathrm{~B}$ \\
\hline Quiina florida & Quinaceae & 0,303 & $\mathrm{C}$ & 0,183 & $\mathrm{C}$ & 0,170 & B \\
\hline Mouriri duckeana & Melastomataceae & 0,418 & $\mathrm{C}$ & 0,186 & $\mathrm{C}$ & 0,170 & B \\
\hline Calyptranthes bipennis & Myrtaceae & 0,288 & $\mathrm{C}$ & 0,181 & $\mathrm{C}$ & 0,169 & B \\
\hline Pouteria filipes & Sapotaceae & 0,379 & $\mathrm{C}$ & 0,182 & $\mathrm{C}$ & 0,169 & $\mathrm{~B}$ \\
\hline Protium tenuifolium & Burseraceae & 0,322 & $\mathrm{C}$ & 0,180 & $\mathrm{C}$ & 0,169 & $\mathrm{~B}$ \\
\hline Helicostylis tomentosa & Moraceae & 0,379 & $\mathrm{C}$ & 0,182 & $\mathrm{C}$ & 0,169 & B \\
\hline Mabea angularis & Euphorbiaceae & 0,327 & $\mathrm{C}$ & 0,179 & $\mathrm{C}$ & 0,169 & $\mathrm{~B}$ \\
\hline Licania heteromorpha & Chrysobalanaceae & 0,344 & $\mathrm{C}$ & 0,180 & $\mathrm{C}$ & 0,169 & B \\
\hline Conceveiba guianensis & Euphorbiaceae & 0,284 & $\mathrm{C}$ & 0,178 & $\mathrm{C}$ & 0,169 & $\mathrm{~B}$ \\
\hline Ocotea petalanthera & Lauraceae & 0,410 & $\mathrm{C}$ & 0,182 & $\mathrm{C}$ & 0,169 & $\mathrm{~B}$ \\
\hline Siparuna amazonica & Monimiaceae & 0,324 & $\mathrm{C}$ & 0,177 & $\mathrm{C}$ & 0,169 & $\mathrm{~B}$ \\
\hline Duroia macrophylla & Rubiaceae & 0,303 & $\mathrm{C}$ & 0,177 & $\mathrm{C}$ & 0,169 & B \\
\hline Pouteria fimbriata & Sapotaceae & 0,400 & $\mathrm{C}$ & 0,179 & $\mathrm{C}$ & 0,169 & B \\
\hline Inga brachystachys & Mimosaceae & 0,268 & $\mathrm{C}$ & 0,175 & $\mathrm{C}$ & 0,169 & $\mathrm{~B}$ \\
\hline Protium opacum ssp. opacum & Burseraceae & 0,304 & $\mathrm{C}$ & 0,175 & $\mathrm{C}$ & 0,169 & $\mathrm{~B}$ \\
\hline Lecythis prancei & Lecythidaceae & 0,376 & $\mathrm{C}$ & 0,178 & $\mathrm{C}$ & 0,168 & B \\
\hline Paypayrola grandiflora & Violaceae & 0,268 & $\mathrm{C}$ & 0,173 & $\mathrm{C}$ & 0,168 & B \\
\hline Pouteria jariensis & Sapotaceae & 0,308 & $\mathrm{C}$ & 0,174 & $\mathrm{C}$ & 0,168 & $\mathrm{~B}$ \\
\hline Eugenia brachypoda & Myrtaceae & 0,255 & $\mathrm{C}$ & 0,171 & $\mathrm{C}$ & 0,168 & $\mathrm{~B}$ \\
\hline Duguetia echinophora & Annonaceae & 0,291 & $\mathrm{C}$ & 0,173 & $\mathrm{C}$ & 0,168 & $\mathrm{~B}$ \\
\hline Cordia umbraculifera & Boraginaceae & 0,293 & $\mathrm{C}$ & 0,170 & $\mathrm{C}$ & 0,168 & B \\
\hline Ocotea cujumary & Lauraceae & 0,279 & $\mathrm{C}$ & 0,170 & $\mathrm{C}$ & 0,168 & B \\
\hline Pouteria reticulata & Sapotaceae & 0,323 & $\mathrm{C}$ & 0,171 & $\mathrm{C}$ & 0,167 & B \\
\hline Guarea grandiflora & Meliaceae & 0,253 & $\mathrm{C}$ & 0,168 & $\mathrm{C}$ & 0,167 & $\mathrm{~B}$ \\
\hline
\end{tabular}


Índice fitossociológico horizontal com variável dummy

A variável dummy (VD), com valor igual a 1, foi atribuída, respectivamente a 46, 29 e 39 espécies que perfizeram mais de $50 \%$ do total das variáveis abundância, frequência e dominância.

A adequação da análise fatorial foi também determinada pelos testes de Bartlett e KMO. O teste de Bartlett avaliou a significância geral da matriz de correlação e apresentou um valor de 3.579,473, indicando que as correlações, em geral, são significantes ao nível de $1 \%$ de probabilidade, ou seja, a matriz de correlação não é diagonal. O teste $\mathrm{KMO}$, da ordem de 0,829 , indicou que as variáveis estão correlacionadas e o modelo fatorial apresentou um nível bom de adequação aos dados (HAIR et al., 2005). Estes resultados respaldam o emprego da análise fatorial para a extração de fatores e a estimação dos escores fatoriais fitossociológicos.

A solução inicial foi rotacionada para os três fatores predeterminados, visando confirmar a adequação dos resultados obtidos com relação à significância das cargas fatoriais estimadas. Aplicando-se o critério da raiz latente, três componentes foram extraídas (DILLON; GOLDSTEIN, 1984). Observou-se que os três fatores explicaram $89,87 \%$ da variância total da nuvem de dados (Tabela 4), o que é amplamente explicado pelo critério da porcentagem da variância. Na referida tabela, a penúltima linha contém o resultado para a variância total explicada por cada uma das componentes, e na última linha estão os percentuais da variância explicada pelas respectivas componentes: a componente 1 explicou $37,29 \%$ da variância total, a componente 2 explicou 26,617\% e a componente 3 explicou 25,963\%.

Tabela 4. Matriz de cargas fatoriais $(\alpha)$ rotacionadas após a rotação ortogonal pelo método varimax com respectivas variáveis dos componentes obtidos (IFH-VD).

Table 4. Matrix of factor loadings $(\alpha)$ rotated after the orthogonal rotation by varimax method with their obtained component variables (IFH-VD).

\begin{tabular}{lcccc}
\hline \multirow{2}{*}{ Variável } & \multicolumn{3}{c}{ Componente } & \multirow{2}{*}{ Comunalidade } \\
\cline { 2 - 4 } & $\mathbf{1}$ & $\mathbf{2}$ & $\mathbf{3}$ & \\
\hline Abundância relativa - ABR & 0,489 & 0,243 & 0,784 & 0,914 \\
Abundância relativa - dummy - ABR-VD & 0,865 & 0,252 & 0,279 & 0,889 \\
Frequência relativa - FRF & 0,626 & 0,309 & 0,632 & 0,887 \\
Frequência relativa - dummy - FRF-VD & 0,860 & 0,214 & 0,289 & 0,869 \\
Dominância relativa - DOR & 0,158 & 0,710 & 0,597 & 0,886 \\
Dominância relativa - dummy - DOR-VD & 0,307 & 0,911 & 0,157 & 0,948 \\
Autovalor & 2,237 & 1,597 & 1,558 & 5,392 \\
Variância explicada (\%) & 37,290 & 26,617 & 25,963 & 89,870 \\
\hline
\end{tabular}

A estatística, detalhando o grau em que cada variável é explicada pelos três componentes, denominada de comunalidade (Tabela 4), na qual a penúltima linha é a soma da coluna de cargas fatoriais ao quadrado (autovalores) e indica a importância relativa de cada fator na explicação da variância associada ao conjunto de variáveis analisadas. A soma de cada fator corresponde aos resultados da coluna quatro da tabela 4, em que o número 5,392 representa a soma total de cargas fatoriais ao quadrado e indica a parcela total de variância extraída pela solução fatorial rotacionada, igual a $89,87 \%$. Ou seja, o índice IFH-VD, para esta solução, mostrou que $89,87 \%$ da variância total são representados pela informação contida na matriz fatorial da solução em termos dos três fatores. $\mathrm{O}$ índice é considerado muito bom, e as variáveis estão, como esperado, estreitamente relacionadas umas com as outras.

O IFH-VD calculado variou de $0,242 \%$ a $0,124 \%$; consequentemente, a amplitude de $0,118 \%$ dividida por 3 definiu uma AR de 0,039\%, gerando os três intervalos das categorias preestabelecidas de predominância das espécies: alta $(0,242 \%$ a $0,203 \%)$, intermediária $(0,202 \%$ a $0,164 \%)$ e baixa $(0,163 \%$ a $0,124 \%$ ). Dessa foram, foram classificadas 29 espécies como de predominância alta, 52 como intermediária e as demais 664 como de baixa predominância (Tabela 2). A relação das 81 espécies classificadas como de alta e intermediária predominância é apresentada na tabela 3.

Índice de Valor de Importância

O IVI calculado variou de $3,408 \%$ a $0,003 \%$, gerando uma amplitude total de $3,405 \%$, que, dividida por 3, resultou numa AR igual a $1,135 \%$, formando os intervalos das três categorias de predominância das espécies: alta (de $3,408 \%$ a 2,273\%), intermediária (de 2,272\% a 1,137\%) e baixa (de $1,136 \%$ a $0,003 \%$ ). 
Foram classificadas apenas quatro espécies na categoria de predominância alta e seis na intermediária; as demais 735 espécies foram classificadas como de predominância baixa (Tabela 2). A relação nominal, com o respectivo valor do índice, de todas as espécies classificadas como de alta e intermediária predominância e das demais 71 espécies com os maiores valores na categoria de baixa predominância é apresentada na tabela 3 .

\section{Comparação entre os índices}

Uma comparação entre os três índices foi efetuada com o intuito de avaliar qual deles incorporava mais informações acerca das espécies, famílias e das associações entre gêneros existentes na fitofisionomia florestal analisada. $\mathrm{O}$ ranqueamento das espécies, feito de forma uniforme para todos os três índices, permitiu visualizar que foram classificadas na categoria de predominância $A$ (alta) 6,29 e 4 espécies, na categoria $B$ (intermediária) 42, 52 e 6 e na $C$ (baixa) 697, 664 e 735, respectivamente, para os índices IFH, IFH-VD e IVI (Tabela 2).

O IVI classificou apenas quatro espécies como de alta predominância (Eschweilera coriacea, Geissospermum sericeum, Rinorea guianensis e Rinorea racemosa), o IFH classificou seis (E. coriacea, G. sericeum, Hevea guianensis, E. amazonica, Minquartia guanensis e Oenocarpus bacaba) e o IFH-VD 29 espécies (Tabela 3). Observou-se que apenas o IFH-VD conseguiu captar, nessa categoria (alta) as importantes associações dos gêneros Pouteria (5 espécies) e Eschweilera (4 espécies), assim como as principais famílias (Sapotaceae, Lecythidaceae, Chrysobalanaceae e Violaceae).

Considerando-se como espécies-chave da comunidade florestal aquelas de predominância ecológica alta e intermediária, foi observado um total de 48, 81 e 10, respectivamente, para o IFH, IFH-VD e IVI (Tabela 2). A relação dessas espécies com os respectivos valores individuais é apresentada na tabela 3.

\section{DISCUSSÃO}

\section{Florística e estrutura}

O trabalho de Cain et al. (1956) marcou o início da utilização, na Amazônia, de parâmetros fitossociológicos, como densidade, frequência, dominância, valor de importância (CURTIS; MCINTOSH, 1951) e da metodologia de caracterização da vegetação de Raunkiaer (1934), que utilizava formas de vida e tamanho da folha.

Um grande problema com que se depara quando se estuda as florestas tropicais e ao mesmo tempo uma grande vantagem que envolve a rica biodiversidade amazônica, refere-se à correta interpretação dos dados. A alta riqueza e diversidade de espécies vegetais presentes na fitocenose analisada, com suas complexas inter-relações, exigem uma grande acurácia na análise. Tome-se como exemplo este trabalho, em que foram registradas 745 espécies, distribuídas em 61 famílias, totalizando 36.546 indivíduos presentes em uma amostra que abrangeu 75,25 ha de Floresta Ombrófila Densa (média

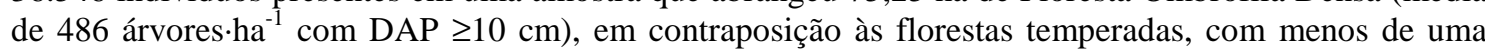
centena de espécies (CURTIS; MCINTOSH, 1951).

A missão da Organização das Nações Unidas para Agricultura e Alimentação (FAO) na Amazônia produziu vários levantamentos florestais na região entre os anos de 1956 e 1961, abrangendo uma faixa de $150 \mathrm{~km}$ de largura por $1.500 \mathrm{~km}$ de comprimento, entre as longitudes $59^{\circ} 30^{\prime} \mathrm{W}$ e $45^{\circ} 30^{\prime} \mathrm{W}$ e as latitudes $2^{\circ} 00^{\prime} \mathrm{S}$ e $6^{\circ} 00^{\prime} \mathrm{S}$ (SUPERINTENDÊNCIA DO DESENVOLVIMENTO DA AMAZÔNIA (SUDAM), 1973). Foram amostrados 1.362 ha (DAP $\geq 25 \mathrm{~cm}$ e em alguns casos DAP $\geq 45 \mathrm{~cm}$ ), onde foram computadas 400 espécies distribuídas em 47 famílias, sendo Leguminosae, Lecythidaceae, Sapotaceae, Burseraceae, Lauraceae e Chrysobalanaceae as de maior riqueza. No presente estudo, as mais representativas foram Leguminosae, com 120 espécies, Sapotaceae (93), Lauraceae (43), Annonaceae (35), Myrtaceae (34), Chrysobalanaceae (33), Burseraceae (30), Moraceae (28) e Lecythidaceae, com 27 espécies, ou seja, todas aquelas famílias de maior riqueza registradas nos estudos da FAO.

Os relatórios da missão informaram que as florestas de terra firme na Amazônia foram separadas segundo dois tipos: associação de espécies do gênero Pouteria e associação de espécies dos gêneros Eschweilera e Pouteria, ambas divididas em 24 fácies ou variações segundo sua composição. No presente estudo, foram identificadas 59 espécies do gênero Pouteria e 13 do gênero Eschweilera, o segundo e o terceiro em abundância no presente trabalho, com, respectivamente, 4.163 e 3.305 árvores, somente superados pelo gênero Rinorea, com 4.277 árvores distribuídas em cinco espécies. Ficou evidenciado 
neste estudo que a cobertura florestal do platô Monte Branco contempla as faciações da Floresta Ombrófila Densa de ambos os gêneros mencionados naqueles relatórios.

Rankin-de-Merona et al. (1992) publicaram os dados preliminares do levantamento de 70 ha de mata de terra firme próximo a Manaus, dentro das reservas do Projeto Dinâmica Biológica de Fragmentos Florestais (PDBFF). Foram computadas 698 morfoespécies, sendo as famílias Lecythidaceae, Leguminosae, Sapotaceae e Burseraceae as mais abundantes em indivíduos e ricas em espécies. O presente estudo corrobora os resultados desses autores. Valencia et al. (1994) amostraram 1 ha $(100 \mathrm{~m} \times 100 \mathrm{~m})$ com a maior alfa-diversidade de árvores do mundo, no Equador, onde foram computadas 473 espécies com DAP $\geq 5 \mathrm{~cm}$ e 307 espécies com pelo menos $10 \mathrm{~cm}$ de DAP. Para essa última classe, Jessenia bataua (Arecaceae) e Eschweilera aff. coriacea (Lecythidaceae) foram as espécies com maior abundância e Leguminosae, Lauraceae, Sapotaceae, Moraceae e Burseraceae as famílias mais diversas. O presente estudo corrobora os dados desses autores.

É muito difícil destacar espécies que, de um modo geral, apresentam uma grande importância na estrutura dessas florestas, sendo Eschweilera coriacea (Lecythidaceae) a única que aparece com grande abundância em praticamente todos os levantamentos na Amazônia (VALENCIA et al., 1994; ROLLET, 1993; SILVA et al., 1992; PRANCE et al., 1976; SUDAM, 1973; CAIN et al., 1956; PIRES et al., 1953, SALOMÃO et al., 2007b). O presente estudo corrobora essa assertiva, uma vez que a referida espécie foi a terceira mais abundante entre as 745 registradas, com 1.271 árvores, que ocorreram em 294 parcelas, ou seja, essa espécie ocorreu em praticamente todas as parcelas $(97,7 \%$ do total) com uma impressionante abundância de 16,9 árvores ha ${ }^{-1}$. Todavia, alguns padrões bem estabelecidos emergem das análises no nível de família: com raras exceções, entre as famílias com maiores abundância e riqueza no dossel de florestas ombrófilas amazônicas estão Leguminosae, Sapotaceae, Lecythidaceae, Burseraceae, Annonaceae, Violaceae, Chrysobalanaceae, Myristicaceae, Lauraceae e Euphorbiaceae.

\section{Comparação entre os índices}

Considerando-se que os resultados referentes às espécies de alta predominância não são suficientes para informar as espécies-chave da fitofisionomia estudada, foi feito o agrupamento das categorias A e B em uma única. Foram então observadas 48, 81 e 10 espécies, respectivamente, para os índices IFH, IFH-VD e IVI nesta nova categoria (A+B) (Tabela 2).

Mesmo com esse procedimento, o IVI não incluiu nenhuma espécie de Pouteria que, como visto, trata-se de um gênero muito importante na caracterização florística das diferentes fácies da floresta amazônica. Por ser um índice que considera tão somente a soma das três variáveis que o formam, todas com o mesmo peso, não conseguiu transmitir uma consistente descrição da composição florística e da estrutura da floresta estudada, através das 10 espécies classificadas como de alta e intermediária predominância ecológica. O IVI, quando proposto por seus autores (CURTIS; MCINTOSH, 1951), foi desenvolvido para florestas temperadas com poucas espécies (25) do sudeste do Wisconsin, nos Estados Unidos. Ao se generalizar o seu emprego para todas as demais fitofisionomias, hão de aparecer as inconsistências de tal decisão. Uma floresta temperada com 25 espécies é completamente diferente de uma floresta tropical amazônica que, como neste caso, apresentou 745 espécies.

Um índice eficaz deve transmitir ao tomador de decisão uma visão realista e o mais sumarizada possível do todo, de tal forma que contenha os principais componentes avaliados, traduzidos em algumas espécies-chave da fitofisionomia em questão, assim como as principais famílias botânicas. O IVI não permitiu visualizar, nas espécies-chave, as famílias características e nem as associações de gêneros descritas por diversos autores para diferentes regiões da Amazônia.

O IFH, obtido através de análise fatorial, pelo método de componentes principais, conseguiu transmitir uma melhor composição das espécies-chave da floresta analisada, por ter considerado as cargas fatoriais ou autovetores que atuaram como ponderações das variáveis consideradas. Apesar das seis espécies de alta predominância serem pouco superiores às do IVI, ao se analisar o agrupamento desta categoria com a da intermediária, observou-se que as 48 espécies resultantes corresponderam a $6,4 \%$ do total das espécies, ou seja, este número é 3,8 vezes superior àquele (Tabela 2).

O IFH-VD, obtido também pelo método de componentes principais, ao considerar uma variável dummy para cada uma das variáveis incluídas no modelo, conseguiu traduzir com muito mais fidelidade a sumarização da composição florística predominante da floresta analisada, permitindo, dessa forma, uma melhor compreensão da complexa estrutura vegetal representada pelas 745 espécies amostradas. As 81 espécies (ou 10,9\% do total) classificadas como de alta e intermediária predominância (Tabela 2), envolveram as famílias, assim como as associações de gêneros, mais características da fitocenose 
estudada. As famílias com maior riqueza e abundância relatadas em diversos estudos em diferentes locais da Amazônia foram captadas por esse índice, destacando-se Sapotaceae (15 espécies), Leguminosae (9), Lecythidaceae (7), Burseraceae e Annonaceae (6 cada) e Chrysobalanaceae e Violaceae (4 cada). As associações de gêneros, intrinsecamente presentes nas diversas facies da floresta amazônica, como Pouteria, Escheweilera Mart. ex DC. e Protium (13, 5 e 5 espécies, respectivamente), foram, também, captadas pelo índice (Tabela 3).

A Secretaria de Meio Ambiente do Estado de São Paulo (SMA) publicou uma legislação inédita para orientar a recuperação de áreas degradadas no Estado: a Resolução SMA 47, de 21/11/2003 (SMA, 2011), que preconiza, entre outras orientações, que nos trabalhos de restauração florestal, em áreas originalmente cobertas por florestas, deverá ser usado um número mínimo de 80 espécies. Observa-se que os resultados do IFH-VD atendem completamente essa exigência. No estado do Pará, o Decreto $\mathrm{n}^{\circ}$ 31.594, de 27/01/2010 (INSTITUTO DE DESENVOLVIMENTO FLORESTAL DO ESTADO DO PARÁ (IDEFLOR), 2010), dispõe no artigo $7^{\circ}$, inciso III, que o plantio de mudas para fins de recomposição da Reserva Legal ( $80 \%$ da área total do imóvel) deverá utilizar o maior número possível de espécies nativas de ocorrência regional.

Conclusivamente, os 22.732 indivíduos $(62,2 \%$ do total) destas 81 espécies de alta e intermediária predominância ecológica, com ampla distribuição na área, observáveis através da inclusão da variável dummy com valor 1, para a quase totalidade das espécies no que se refere à abundância e frequência, e cerca de ${ }^{2} / 3$ das espécies para a variável dominância, mostraram que o IVI é o mais eficiente índice para uma análise da fitossociologia horizontal das espécies.

No entanto, há de se frisar que todas as espécies são únicas e relacionadas entre si no complexo ecossistema florestal amazônico. Ao se procurar caracterizar as espécies-chave de um determinado ambiente, não se pode excluir da análise as demais, pois, como se sabe, cerca de $1 / 5$ a $1 / 3$ das espécies ocorrem com apenas um indivíduo na totalidade dos levantamentos executados em florestas primárias na Amazônia.

Salomão et al. (2007a) ressaltaram que, no platô Monte Branco, foi observada a ocorrência de espécies raras na Amazônia, como por exemplo Micrandropses scleroxylon W. A. Rodrigues, Micrandra rossiana R. E. Schultes, Duckeodendron cestroides Kuhlm., Copaifera reticulata Ducke, Spiranthera guianensis Sandwith, Pouteria laevigata (Mart.) Radlk., Roupala obtusata Klotzsch, Tabebuia impetiginosa (Mart.) Standl. e Sterigmapetalun obovatum Kuhlm. Também nunca haviam sido registradas com elevada abundância, nos demais platôs da Flona Saracá-Taquera/ICMBio, as espécies Copaifera reticulata e Micrandropses scleroxylon.

\section{CONCLUSÃO}

- Os testes estatísticos utilizados permitiram testar a adequação da amostra de dados para o emprego da técnica de análise multivariada e, portanto, para a construção dos índices IFH-VD e IFH.

- Os índices propostos (IFH-VD e IFH) permitiram selecionar um percentual bem superior de espécies-chave do que o IVI que, como era esperado, se mostrou menos apropriado para a indicação de espécies-chave do que os índices ora propostos, em função do reduzido número de espécies obtido por meio do ranqueamento nas categorias de predominância alta e intermediária.

- A indicação dos índices propostos neste trabalho como metodologia alternativa, em função do rigor científico, deve merecer aplicações em outras fitofisionomias para efeito de validação, uma vez que torna a seleção de espécies-chave mais abrangente e adequada do que pelo IVI.

- A restauração florestal de áreas degradadas, especialmente aquelas situadas em unidades de conservação, deve ser orientada por princípios técnicos e científicos mais rígidos, no sentido de propiciar ao novo ecossistema artificial criado a presença e abundância de espécies que atendam a alguns indispensáveis parâmetros ecológicos mínimos observados na cobertura florestal original.

- Os índices propostos podem contribuir para as políticas públicas que visam a recuperação de áreas degradadas pelas atividades minerárias e, também, para a restauração de APP ou RL - passivo ambiental - fortemente antropizadas pela substituição de florestas, com base na indicação de espécies-chave mais adequadas para o plantio visando restaurar a paisagem florestal primitiva.

\section{REFERÊNCIAS}

CAIN, S. A.; CASTRO, G. M. A.; PIRES, J. M.; SILVA, N. T. Application of some phytosociological techniques to brazilian rain forest. American Journal of Botany, St. Louis, v. 43, p. 911 - 941, 1956. 
CAMPOS, J. C. C.; LEITE, H. C. Mensuração florestal: perguntas e respostas. Viçosa: Universidade Federal de Viçosa, 2002. 407 p. (in Portuguese)

COSTA, N. A. A. A reciclagem de resíduo de construção e demolição: uma aplicação de análise multivariada. 188 p. Tese (Doutorado em Engenharia da Produção) - Universidade Federal de Santa Catarina, Florianópolis, 2003.

CRONQUIST, A. An integrated system of classification of flowering plants. New York: Columbia University, 1981. $555 \mathrm{p}$.

CURTIS, J. T.; MCINTOSH. An upland forest continuum in the prairie-forest border region of Wisconsin. Ecology, Washington, v. 32, p. 476 - 496, 1951.

DILLON, W. R.; GOLDSTEIN, M. Multivariate analysis. Methods and applications. New York, John Wiley and Sons, 1984. 496 p.

GAMA, Z. J. C.; SANTANA, A. C. DE; MENDES, F. A. T.; KHAN, A. S. Índice de desempenho competitivo das empresas de móveis da Região Metropolitana de Belém. Revista de Economia e Agronegócio, Rio de Janeiro, v. 5, p. 127 - 159. 2007.

GAUCH, H. G. Multivariate Analysis in Community Ecology. Cambridge Univ. Press. New York. 1982. $298 \mathrm{p}$.

GOODALL, D. W. Objective methods for the classification of vegetation. III. An essay in the use of factor analysis. Australian Journal of Botany, Collingwood, v. 2, p. 304 - 324. 1954.

HAIR JR., J. F.; ANDERSON, R. E.; TATHAM, R. L.; BLACK, W. C. Análise multivariada de dados. 5. ed. Porto Alegre: Bookman, 2005. 593 p.

INSTITUTO DE DESENVOLVIMENTO FLORESTAL DO ESTADO DO PARÁ (IDEFLOR), Instrução Normativa $\mathbf{n}^{\mathbf{0}}$ 02/2010. Disponível em: <(http://www.ideflor.pa.gov.br/files/u3/IN022010.pdf)>. Acesso em: 18/10/2010.

INSTITUTO NACIONAL DE METEOROLOGIA (INMET) Disponível em: <http://www.inmet. gov.br/html/clima/mapas/?mapa=inso >. Acesso em: 12/06/2006.

JOHNSON, R. A.; WICHERN, D. W. Applied multivariate statistical analysis. 3. ed. New Jersey: Prentice-Hall, 1992. 642 p.

KAPPELLE, M. K.; DENNIS, P. A. F.; de VRIES, R. A. J. R. Changes in diversity along a successional gradient in a Costa Rica upper montane. Biodiversity and Conservation, Nairobi, v. 4, p. 10 - 34, 1995.

KÖPPEN, W.; GEIGER, R. Klimate der Erde. Gotha: VerlagJustus Perthes, 1928.

LA ROI, G. H. Classification and ordination of southern boreal forest form the Hondo - Slave Lake area of central Alberta, Canada. Journal of Botany, Edinburg, v. 70, p. 614 - 628, 1992.

LAPA, R. P. A bauxita e o rejeito da bauxita. In: BOZELLI, R. L.; ESTEVES, F. A.; ROLAND, F. Lago Batata: Impacto e Recuperação de um Ecossistema Amazônico. Rio de Janeiro, 2000. p. 27 - 35.

MINGOTI, S. A. Análise de dados através de métodos de estatística multivariada: uma abordagem aplicada. Belo Horizonte: Editora Universidade Federal de Minas Gerais, 2005. 279 p.

OGUTU, Z. A. Multivariate analysis of plant communities in Narok district, Kenya: the influence of environmental factors and human disturbance. Vegetation, Bruxelle, v. 126, p. 181 - 189, 1996.

OLIVEIRA-FILHO, A. T.; FONTES, M. A. L. Patterns of floristic differentiation among Atlantic Forests in Southeastern Brazil and the influence of climate. Biotropica, Zurich v. 32, p. 793 - 810, 2000.

OLIVEIRA-FILHO, A. T.; RATTER, J. A. A study of the origin of central Brazilian forests by the analysis of plant species distribution patterns. Edinburgh. Journal of Botany, Edinburg, v. 52, p. 141 194, 1995.

OLIVEIRA-FILHO, A. T.; VILLELA, E. A.; GAVILANES, M. L.; CARVALHO, D. A. Comparison of the Woody flora and soils of six areas of montane semiciduous Forest in southern Minas Gerais, Brazil. Edinburgh. Journal of Botany, Edinburg, v. 513, p. 355 - 389, 1994.

PIELOU, E. C. The interpretation of ecological data: a primer on classification and ordination. WileyInterscience Publ. New York, 1984. 263 p.

PIRES, J. M.; DOBZANSKY, T.; BLACK, G. A. An estimate of the number of species of trees in an 
Amazonian Forest Community, Botanical Gazette, New York, v. 114, p. 467 - 477, 1953.

PRANCE, G. T.; RODRIGUES, W. A.; SILVA, M. F. Inventário florestal de um hectare de mata de terra firme, km 30 da estrada Manaus-Itacoatiara. Acta Amazônica, Manaus, v. 6, p. 9 - 35, 1976.

PROJETO RADAM. Folha SA21 Santarém. Ministério das Minas e Energia: Departamento Nacional de Produção Mineral, Rio de Janeiro, 1976, v. 10, p. 309 - 414.

RANKIN-DE-MERONA, J. M.; PRANCE, G. T.; HUTCHINGS, R. W.; SILVA, M. F.; RODRIGUES, W. A.; UEHLING, M. E. Preliminar results of a large-scale tree inventory of upland rain forest in the central Amazon. Acta Amazônica, Manaus, v. 22, p. 493 - 534, 1992.

RAUNKIAER, C. The life forms of plants and statistical geography. Clarendon Press. Oxford. 1934. $632 \mathrm{pp}$.

RODRIGUES, R. R.; GANDOLFI, S. Recomposição de florestas nativas: princípios gerais e subsídios para uma definição metodológica. Revista Brasileira de Horticultura e Plantas Ornamentais, Campinas, v. 2, p. 4 - 15, 1996.

ROLLET, B. Tree populations in natural tropical rain forest. Bois et Forêsts des Tropiques, Montpellier, v. 236, p. $43-55,1993$.

SALOMÃO, R. P.; ROSA, N. A.; MORAIS, K. A. C. Dinâmica da regeneração natural de árvores em áreas mineradas da Amazônia. Boletim do Museu Paraense Emílio Goeldi, Ciências Naturais, Belém, v. 2, p. 85 - 139, 2007a.

SALOMÃO, R. P.; VIEIRA, I. C. G.; SUEMITSU, C.; ROSA, N. A.; ALMEIDA, S. S.; AMARAL, D. D.; MENEZES, M. P. M. As florestas de Belo Monte na grande curva do rio Xingu, Amazônia oriental.

Boletim do Museu Paraense Emílio Goeldi, Ciências Naturais, Belém, v. 2, p. 57 - 153, 2007b.

SANTANA, A. C. Análise do desempenho competitivo das agroindústrias de polpa de frutas do Estado do Pará. Teoria e Evidência Econômica, Passo Fundo, v. 14, p. 36 - 62, 2007.

SANTANA, A. C. de. Elementos de economia, agronegócio e desenvolvimento local. GTZ; TUD; Universidade Federal Rural da Amazônia. Série Acadêmica, Belém, v. 1, p. 133 - 142, 2005.

SANTANA, A. C. de; SANTANA, A. L. de. Mapeamento e análise de arranjos produtivos locais na Amazônia. Teoria e Evidência Econômica, Passo Fundo, v. 12, p. 9 - 14, 2004.

SANTANA, A. C. Índice de desempenho competitivo das empresas de polpa de frutas do Estado do Pará. Análise sistêmica da fruticultura paraense: organização, mercado e competitividade empresarial. 1 ed. Belém - PA: Banco da Amazônia, Belém, v. 1, p. 115 - 143, 2008.

SCUDELLER, V. V.; MARTINS, F. R.; SHEPHERD, G. J. Distribution and abundance of arboreal species in the atlantic ombrophilous dense forest in Southeastern Brazil. Plant Ecology, New York, v. 152, p. 185 - 199, 2001.

SILVA, A. S. L.; LISBOA, P. L. B.; MACIEL, U. N. Diversidade florística e estrutura em Floresta Densa da bacia do Rio Juruá. Boletim do Museu Paraense Emílio Goeldi, Série Botânica, v. 8, n. 2, p. 203 $258,1992$.

SECRETARIA DE MEIO AMBIENTE DO ESTADO DE SÃO PAULO (SMA). Resolução SMA 43, de 21/11/2003. Diponível em http://sigam.ambiente.sp.gov.br/Sigam2/legisla\%C3\%A7\%C3\%A3o\% 20ambiental/resolu\%C3\%A7\%C3\%A3o\%20sma\%202003_047.pdf. Acesso em: 04/06/2011.

SUPERINTENDÊNCIA DE DESENVOLVIMENTO DA AMAZÔNIA (SUDAM). Levantamentos florestais realizados pela missão FAO na Amazônia. Superintendência do Desenvolvimento da Amazônia, Belém, 1973. v. 2, 397 p.

TAGGART, J. B. Ordination as an aid in determining priorities for plant community protection. Conservation Biology, Washington, v. 68, p. 135 - 141, 1994.

TOLEDO, L. de O.; ANJOS, L. H. C.; COUTO, W. H.; CORREIA, J. R.; PEREIRA, M. G.; CORREIA, M. E. F. Análise multivariada de atributos pedológicos e fitossociológicos aplicada na caracterização de ambientes de cerrado no norte de Minas Gerais. Revista Árvore, Viçosa, v. 33, n. 5, p. 955 - 966, 2009.

VALENCIA, R.; BALSLEV, H.; PAZ Y MINO, G. C. High tree alpha-diversity in Amazonian Ecuador. Biodiversity and Conservation, New Zealand, v. 3, p. 21 - 28, 1994. 